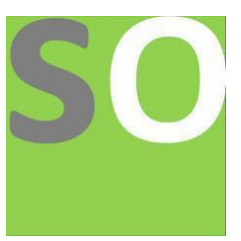

Article title: Analysis of Friction, Spring and Wedging action

Authors: Bo-Göran Wallner[1]

Affiliations: No affiliation[1]

Orcid ids: 0000-0002-7713-4475[1]

Contact e-mail: bogoeran.wallner@protonmail.ch

License information: This work has been published open access under Creative Commons Attribution License http://creativecommons.org/licenses/by/4.0/, which permits unrestricted use, distribution, and reproduction in any medium, provided the original work is properly cited. Conditions, terms of use and publishing policy can be found at https://www.scienceopen.com/.

Preprint statement: This article is a preprint and has not been peer-reviewed, under consideration and submitted to ScienceOpen Preprints for open peer review.

DOI: 10.14293/S2199-1006.1.SOR-.PPXEO6V.v3

Preprint first posted online: 20 February 2021 


\title{
Analysis of Friction, Spring and Wedging action ${ }^{\star \star \star \star}$
}

\author{
EUR ING B.G. Wallner ${ }^{\mathrm{a}, 1}$, \\ ${ }^{a}$ Mistelvaegen 13, 244171 Doesjebro, Sweden, +46732484998
}

\begin{abstract}
In this educational academic paper we will derive the equations of motion for a wedge in contact by friction with spherical object. This object is connected to a spring. We will approach the problem with Newtonian mechanics and compare this result with a Lagrangian approach and we will show that they with some restriction lead to the same result. The results from the equations of motion will be discussed. The equations will be numerically simulated for a number of different cases and the results will be analyzed.
\end{abstract}

Keywords: Classical mechanics, friction, machine element, wedge, spring

\section{Introduction}

The following idealized problem was introduced within the industry for determining a spring constant for a given physical situation. We won't go into the exact details of the application but the appearance of simple problems might sometimes reveal interesting properties. We will stress the importance of understanding the difference between mathematical model and reality. Why does an object fall? One could argue it's because of Newton's law of gravitation. Well, of course the intention is correct but even though, it might be an indication of how we sometimes mix up nature with the mathematical model. The answer is because that's the way nature works. The Newtonian and Euler-Lagrange's equation are mathematical models describing and predicting the motion of an object or system of objects, valid under certain circumstances. And we will investigate some of these circumstances in the following discussion. The discussion in this paper far exceeds what is acctually needed for the solution of the original problem.

\section{Analysis}

Let's start this study and consider the following setup.

\footnotetext{
${ }^{\star}$ Or the art of writing a lot about almost nothing.

${ }^{\star}$ Devoted to Arne Ekstrand, teacher in Teknologi at Pauliskolan during the 1990ths. Email address: bogoeran.wallner@gmail.com (EUR ING B.G. Wallner)

${ }^{1}$ Master of Science in Engineering Physics, Lund university, Faculty of Engineering.
} 


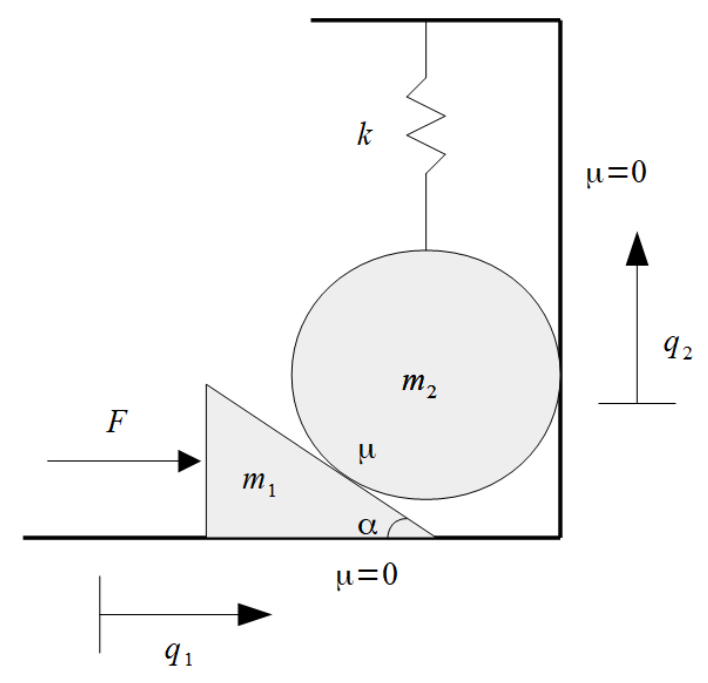

The setup have two different moving elements, a spherical object with mass $m_{2}$ connected to a spring defined by the constant $k$. The spherical object is in contact with a wedge by a constant friction $\mu$ and two coordinates $q_{1}$ and $q_{2}$ are introduced. There is no rolling motion by the spherical object, only a sliding motion. The coordinates should be understood as $q_{1}=q_{2}=0$ when the spring is initially uncompressed. The result would basically be the same if the spring was initially loaded but leads to no further conclusions about the problem. The wedge having the mass $m_{1}$ is pushed by a constant force $F$. The friction at the bottom and side is considered as $\mu=0$. We might also interpret it as the friction forces arising from these are small in comparison to $F$ and the spring force. Intuitively, we understand that when $F$ is applied to the wedge it causes the spherical object to rise and the spring to compress until a steady state is reached, given that $F$ is of reasonable size. And during this study, we restrict ourselves to this one way motion of compressing the spring to avoid problems with a changing sign of the friction force. One of the challenges when dealing with friction forces is to understand the direction in which they act, understanding that a friction force counteracts the direction of the movement. If instead an energy approach, such as Euler-Lagrange's equation is used, it's more obvious that there is a loss of energy when the virtual work done by the friction forces are converted into heat. The direction of the friction forces might not in all cases be very obvious if the geometry is complicated. In this problem one would benefit from starting to write down the equations of motion for the wegde, we will however start with the spherical object.

\subsection{Newton's approach}

In this section we will derive the equations of motion for the two objects, the wedge and the spherical object using the relation 


$$
\overrightarrow{F_{\text {ext }}} \cdot \frac{1}{m}=\left(\sum_{i} \overrightarrow{F_{i}}\right) \cdot \frac{1}{m}=\vec{a}
$$

Or as we usually write down the equations of motion

$$
\vec{F}_{\text {ext }}=m \cdot \vec{a}
$$

One can wonder about the former notation, one might argue that it better reflects cause and action.

First we write down a free-body diagram of all forces acting upon the wedge and the spherical object.

"Half of the problem is solved when a good picture has been drawn".

-Arne Ekstrand
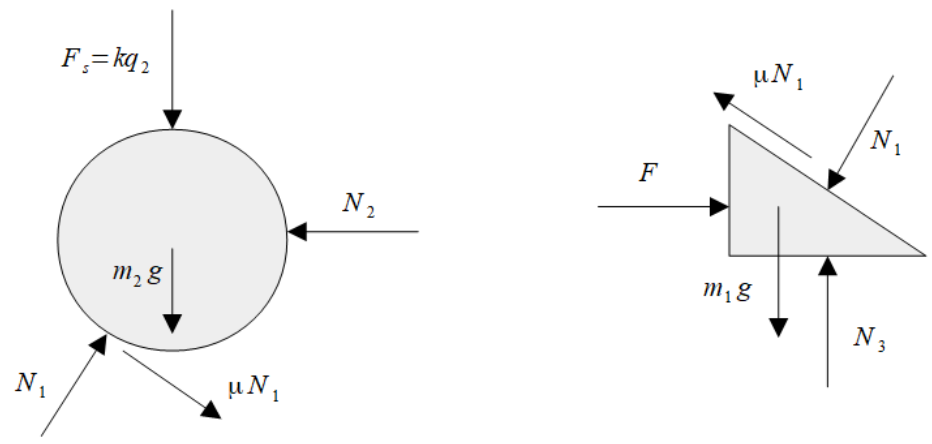

Starting with the spherical object the force from the spring $F_{s}$ act on the object counteracting the upward motion. From the wall the normal force $\mathrm{N}_{2}$ acts and besides the gravitation acting on the mass $m_{2}$ the normal force $N_{1}$ acts on it from the wedge. Now, we turn our attention to the friction force which is assumed to be a constant Coloumb friction. The only absolute motion done by the spherical object is upwards, however, there is a relative motion against the wedge and seen from the reference frame of the wedge, the spherical object is moving upwards. Thus the friction force shall be directed opposite to this upward motion. If we instead started by analyzing the wedge, the direction of the friction force acting upon it would be more obvious.

$$
\begin{array}{r}
\uparrow-k \cdot q_{2}-m_{2} g+N_{1} \cos (\alpha)-\mu N_{1} \sin (\alpha)=m_{2} \ddot{q}_{2} \\
\rightarrow N_{1} \sin (\alpha)+\mu N_{1} \cos (\alpha)-N_{2}=0
\end{array}
$$


Rearranging the equations

$$
\begin{aligned}
\uparrow m_{2} \ddot{q_{2}}+k \cdot q_{2} & =N_{1}(\cos (\alpha)-\mu \cdot \sin (\alpha))-m_{2} g \\
& \rightarrow N_{2}=N_{1}(\sin (\alpha)+\mu \cdot \cos (\alpha))
\end{aligned}
$$

The equations for the wedge can be written as

$$
\begin{gathered}
\uparrow N_{3}-m_{1} g-N_{1} \cos (\alpha)+\mu N_{1} \sin (\alpha)=0 \\
\quad \rightarrow F-N_{1} \sin (\alpha)-\mu N_{1} \cos (\alpha)=m_{1} \ddot{q}_{1} \\
\rightarrow N_{1}=\frac{F-m_{1} \ddot{q}_{1}}{\sin (\alpha)+\mu \cdot \cos (\alpha)}=k_{1}\left(F-m_{1} \ddot{q}_{1}\right) \\
k_{1}=\frac{1}{\sin (\alpha)+\mu \cdot \cos (\alpha)}
\end{gathered}
$$

Solving for $N_{3}$ does not provide us with any more useful information for determining the motion. One thing we can notice is that we connect the two parts, the wedge and the spherical object through the force $N_{1}$. Since we now have an expression for $N_{1}$ we can use this in (1).

$$
\uparrow m_{2} \ddot{q}_{2}+k \cdot q_{2}=k_{1}\left(F-m_{1} \ddot{q}_{1}\right)(\cos (\alpha)-\mu \cdot \sin (\alpha))-m_{2} g
$$

Previously we said that we have two coordinates $q_{1}$ and $q_{2}$ and we understand that they are connected through geometry. Consider the spherical object, represented by the moving primed coordinate system, at time $t$ and $t+\Delta t$ according to the image below.

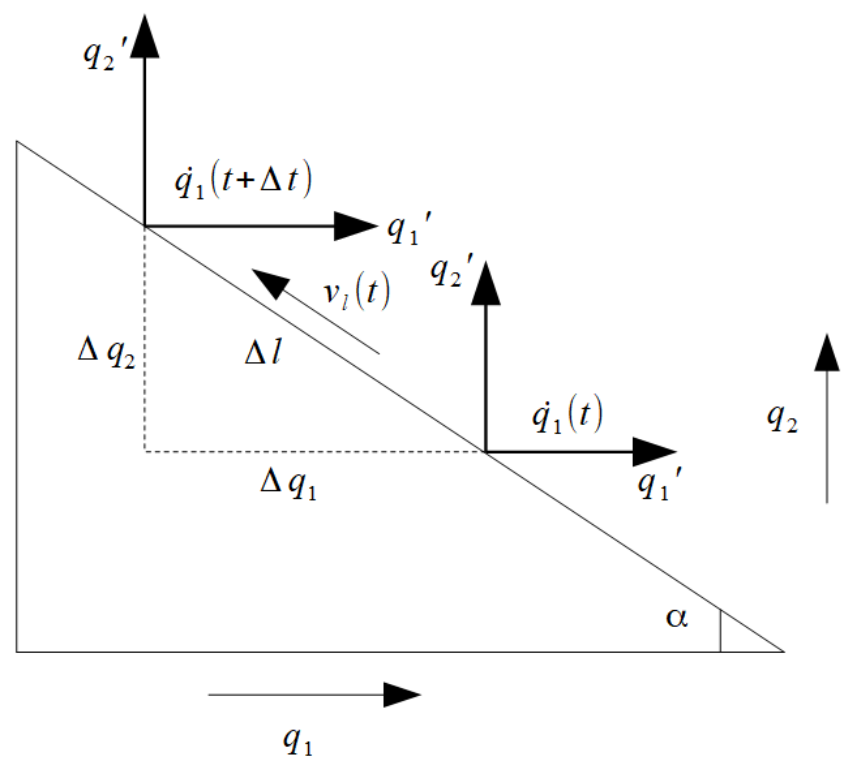


From the image we can conclude that the pure geometrical relation between $\Delta q_{1}$ and $\Delta q_{2}$ is

$$
\Delta q_{2}=\Delta q_{1} \tan (\alpha)
$$

Equation (5) have derivatives of both $q_{1}$ and $q_{2}$ which needs to be sorted out. In the image above let's assume that the wedge is fixed and that the spherical object moves up along the inclination. The velocity of the spherical object in the negative $q_{1}$ direction at a time $t$ is $\dot{q}_{1}(t)$. During the time $t$ and $t+\Delta t$ the spherical object have moved the distance $\Delta l$ with the velocity $v_{l}(t)$.

$$
v_{l}(t) \approx \frac{\Delta l}{\Delta t}=\frac{q_{1}(t+\Delta t)-q_{1}(t)}{\cos (\alpha)} \cdot \frac{1}{\Delta t}
$$

This velocity can be decomposed into the $q_{1}$ and $q_{2}$ direction which gives us for the vertical motion

$$
\frac{\Delta q_{2}}{\Delta t}=\frac{\Delta l}{\Delta t} \cdot \sin (\alpha)=\frac{q_{1}(t+\Delta t)-q_{1}(t)}{\Delta t} \cdot \frac{1}{\cos (\alpha)} \cdot \sin (\alpha)
$$

From this result we can conclude that we finally get the relations when letting $\Delta t \rightarrow 0$

$$
\dot{q}_{2}=\dot{q}_{1} \tan (\alpha) \rightarrow \ddot{q_{2}}=\ddot{q}_{1} \tan (\alpha)
$$

We can also notice, not suprisingly, that we could get this result directly from the geometrical relation (6). One could oppose to the somewhat sloppy handling concerning the sign but since we are engineers and not physicists we can get away with it.

Using this result in (5) gives us

$$
\uparrow m_{2} \ddot{q_{2}}+k \cdot q_{2}=\left(k_{1} F-\frac{k_{1} m_{1}}{\tan (\alpha)} \cdot \ddot{q}_{2}\right)(\cos (\alpha)-\mu \cdot \sin (\alpha))-m_{2} g
$$

Let us simplify by substituting

$$
k_{2}=\cos (\alpha)-\mu \cdot \sin (\alpha)
$$

which then gives us

$$
\uparrow m_{2} \ddot{q}_{2}+k \cdot q_{2}=k_{1} k_{2} F-\frac{k_{1} k_{2} m_{1}}{\tan (\alpha)} \cdot \ddot{q_{2}}-m_{2} g
$$

and gathering all coordinate dependence on one side finally gives

$$
\begin{array}{r}
\uparrow\left(m_{2}+\frac{k_{1} k_{2}}{\tan (\alpha)} \cdot m_{1}\right) \cdot \ddot{q}_{2}+k \cdot q_{2}=k_{1} k_{2} F-m_{2} g \\
k_{1} k_{2}=\frac{\cos (\alpha)-\mu \cdot \sin (\alpha)}{\sin (\alpha)+\mu \cdot \cos (\alpha)}
\end{array}
$$


Noticing that $k_{1}$ and $k_{2}$ are dimensionless we can conclude that all entities have the same unit. Solving this equation will fully determine the motion of the system. One interesting observation we can do is the expression $k_{1} k_{2}$ which can be seen in books covering theory of machine elements [Ref 1], translating an axial load into a wedging force. Also similarities to screws can be noticed [Ref 2]. Applications might be e.g. conical rings attaching a bearing to an axle.

\subsection{A Lagrangian approach}

The approach to solve the problem with analytical mechanics and EulerLagrange's equation will be discussed here. We will approach the problem without considering the friction. The formulation of Euler-Lagrange's equation requires a set of independent generalized coordinates $\left(q_{0}, q_{1}, q_{2}, \ldots, q_{n}\right)$ where $q_{i}$ is independent of $q_{j}$. Previously we have introduced variables called $q_{1}$ and $q_{2}$ based upon our understanding about how the system evolves. But let's first look at the formulation of Euler-Lagrange's equation.

The reader will find no figures in this work. The methods which I set forth do not require either constructions or geometrical or mechanical reasonings: but only algebraic operations, subject to a regular and uniform rule of procedure. -Joseph-Louis Lagrange

The Euler-Lagrange's equation can derived from D'Alembert's principle and for systems where forces can be derived from a potential $F_{i}=-\nabla_{i} V$ the equation can be written as

$$
\frac{d}{d t}\left(\frac{\partial L}{\partial \dot{q_{j}}}\right)-\frac{\partial L}{\partial q_{j}}=0
$$

where $L(q, \dot{q}, t)=L=T-U$ is called the Lagrangian for the system and describes the difference between kinetic energy and potential energy. It's important to stress that the Lagrangian describes the whole system and not parts of it, and for each generalized coordinate $q_{j}$ we get one equation. As stated this equation describes a system where the forces can be derived from a potential. In our case that is not true for the externally applied force $F$ which is acting on the wedge. Nor is it the case for the friction. However, this can be accounted for by modifying the equation to [Ref 3$]$

$$
\frac{d}{d t}\left(\frac{\partial L}{\partial \dot{q}_{j}}\right)-\frac{\partial L}{\partial q_{j}}=Q_{j}
$$

where $Q_{j}$ is a generalized force defined according to

$$
Q_{j}=\sum_{i} \overrightarrow{F_{i}} \cdot \frac{\partial \overrightarrow{r_{i}}}{\partial q_{j}}
$$


Since the choice of generalized coordinates are important for the formulation of the equations we will discuss this some more. First let's assume that the positions of the objects are described in a cartesian coordinate system with the vectors $\overrightarrow{r_{1}}=\left(x_{1}, y_{1}\right)$ and $\overrightarrow{r_{2}}=\left(x_{2}, y_{2}\right)$. If these were chosen as generalized coordinates we would require four Lagrangian equations. With a suitable choice of the origin we could make sure that $y_{1}=0$ (wedge) and that $x_{2}-c=0$ (spherical object) for some constant $c$. If these were generalized coordinates we could now describe the system by only two Lagrangian equations for $x_{1}$ and $y_{2}$. However, as we have seen $x_{1}$ and $y_{2}$ are not independent but connected through another constraint stating that $\Delta y_{2}=\Delta x_{1} \cdot \tan (\alpha) \Leftrightarrow \Delta y_{2}-\Delta x_{1} \cdot \tan (\alpha)=$ 0 . What this means is that we can reduce the number of equations from the previous two equations to only one. A holonomic constraint is a constraint such that there exist a function $f\left(\overrightarrow{r_{1}}, \overrightarrow{r_{2}}, \ldots, t\right)=0$ with which we can describe the constraint. As we have seen above, this is exactly what has been done. Thus, we can choose e.g. $q_{2}$ as generalized coordinate. So we have approached this result from a little bit different perspective than the purely geometrical discussion above in the Newtonian view.

The kinetic energy of the system can be written as

$$
T=\frac{m_{1} \dot{q}_{1}^{2}}{2}+\frac{m_{2} \dot{q}_{2}^{2}}{2}=\frac{m_{1} \dot{q}_{2}^{2}}{2 \cdot \tan ^{2}(\alpha)}+\frac{m_{2} \dot{q}_{2}^{2}}{2}
$$

The potential energy from the gravitational force and spring force

$$
U=\frac{k \cdot q_{2}^{2}}{2}+m_{2} g \cdot q_{2}
$$

Which then gives us the Lagrangian

$$
L=\frac{m_{1} \dot{q_{2}^{2}}}{2 \cdot \tan ^{2}(\alpha)}+\frac{m_{2} \dot{q}_{2}^{2}}{2}-\frac{k \cdot q_{2}^{2}}{2}-m_{2} g \cdot q_{2}
$$

Inserting this into (9) gives us

$$
\begin{array}{r}
\frac{d}{d t}\left(\frac{\partial L}{\partial \dot{q}_{j}}\right)-\frac{\partial L}{\partial q_{j}} \\
=\frac{d}{d t}\left(\frac{m_{1} \dot{q_{2}}}{\tan ^{2}(\alpha)}+m_{2} \dot{q_{2}}\right)+k \cdot q_{2}+m_{2} g= \\
=\left(m_{2}+\frac{m_{1}}{\tan ^{2}(\alpha)}\right) \cdot \ddot{q}_{2}+k \cdot q_{2}+m_{2} g=Q_{2}
\end{array}
$$

For $Q_{2}$ we can use (10)

$$
Q_{2}=\sum_{i} \vec{F}_{i} \cdot \frac{\partial \overrightarrow{r_{i}}}{\partial q_{2}}=\frac{F}{\tan (\alpha)}
$$

To be able to understand this result consider the virtual work done on the wedge by the force $F$ according to $\delta W_{1}=F \cdot \delta q_{1}$. The output from the system, the work done by the wedge, can be written $\delta W_{2}=Q_{2} \cdot \delta q_{2}=Q_{2} \cdot \delta q_{1} \cdot \tan (\alpha)$. The amount of energy put into the system is equal to the amount of energy taken 
out from the system according to conservation of energy, thus $\delta W_{1}=\delta W_{2}$ and the result for $Q_{2}$ follows. What is gained in force we loose in distance. Looking at the limiting cases is the second thing that an engineer do after checking that the units are correct. We see that for $\alpha=0$ (flat wedge) we get a force $Q_{2} \rightarrow \infty$ but at the same time we must have $\delta q_{2} \rightarrow 0$ (otherwise leading to infinite energy). The same reasoning applies for $\alpha=\frac{\pi}{2}$ where $Q_{2} \rightarrow 0$ and $\delta q_{2} \rightarrow \infty$. This agrees with intuition. Finally we can then write down the full equation

$$
\left(m_{2}+\frac{m_{1}}{\tan ^{2}(\alpha)}\right) \cdot \ddot{q}_{2}+k \cdot q_{2}=\frac{F}{\tan (\alpha)}-m_{2} g
$$

If we now look at the Newtonian result in (8) and let the friction $\mu=0$ we get $k_{1} k_{2}=\frac{\cos (\alpha)}{\sin (\alpha)}=\frac{1}{\tan (\alpha)}$. Using this in (7)

$$
\begin{aligned}
& \left(m_{2}+\frac{k_{1} k_{2}}{\tan (\alpha)} \cdot m_{1}\right) \cdot \ddot{q}_{2}+k \cdot q_{2}=k_{1} k_{2} F-m_{2} g \rightarrow \\
& \quad \rightarrow\left(m_{2}+\frac{m_{1}}{\tan ^{2}(\alpha)}\right) \cdot \ddot{q_{2}}+k \cdot q_{2}=\frac{F}{\tan (\alpha)}-m_{2} g
\end{aligned}
$$

We have verified that the two different approaches lead to the same equation. So what about the friction. In the Newtonian approach we have chosen to model the friction as a Coloumb friction which is expressed as $F_{f}=\mu \cdot N_{\text {normal }}$. This model for the friction requires us to calculate the normal force between the wedge and the spherical object but when choosing the Lagrangian approach the goal is to avoid dealing with forces. Thus, there is a need for another friction model in the Lagrangian approach and one option is to use the Rayleigh's dissipation function which is discussed in [Ref 3] [Ref 4]. However, another model would lead to other results and we would not be able to directly compare the different mathematical models. We now stop this analysis and turn over to some concluding remarks for the analysis.

\subsection{Concluding remarks}

In this section we will briefly review the analysis made. The main problem with the Newtonian approach was to be able to determine the direction of the friction force and we also needed to think about the relative movement between the two objects. The choice of which object to start to analyze turned out to have been easier if we chose the object acted upon by the force $F$ (the wedge) rather than the spherical object having a relative motion in the $q_{1}$ direction. We also needed to add forces from constraints when fully writing down the equations, i.e. $N_{3}$ and $N_{2}$. However, we could just ignore these forces based upon physical reasoning. We then used the geometrical relation to connect $q_{1}$ with $q_{2}$ and then extended this to the derivatives. For the Lagrangian approach we started of by discussing generalized coordinates and holonomic constraints with some more formalism and we concluded that we could use only generalized coordinate. This reduced a problem described in four cartesian coordinates to be solved with only one Lagrangian equation. We discussed the generalized 
force $Q j$ arising from $F$ and showed by the principle of virtual work how we could find a relation between them. During the simplification of this equation we ignored the friction, which would otherwise be added to $Q j$, and we showed that the result was exactly the same as with the Newtonian approach with the friction $\mu=0$. We also concluded that if we wanted to include friction in the Lagrangian approach we needed another model for the friction not relying upon internal forces between objects. In the next section we will review the properties of this equation. It's already now time to mention, this equation describes an infinite set of solutions, most of them are not physically relevant. Only a certain combination of $m_{1}, m_{2}, F, \ldots$ will generate a solution which acctually describes the reality, given the assumptions made in the mathematical model itself. And even if we choose the correct parameters which will generate a valid solution, it will only be physically relevant for parts of it, for example $q_{2} \geq 0: \forall t$.

\section{Simulation \& results}

In this section we will look at some of the results from simulating the equation (7) by varying the different parameters and see how they change the result. To be able to simulate this in MATLAB we need to choose a set of parameters and this is done by choosing parameters in such a way that it illustrates some of the properties. As we have discussed before we need to be aware that this is a mathematical model describing the nature under certain circumstances and we must be able to interpret the results by engineering intuition. The values chosen are not typically values for mechanical engineering.

\subsection{Simulation of dynamics}

In all simulations the force $F=100 N$ is used, masses are between $m=$ $0.5 \ldots 2 \mathrm{~kg}$ and the spring constant $k=5 \mathrm{~N} / \mathrm{m}$. This will make large compressions of the spring and the size of the wedge should have to be of unrealistic size. But these shortcomings are just a matter of scaling. Let's look at the first simulation.

\subsubsection{Impact of variation in mass}

The results from the simulation can be seen in Figure 1.

In this graph we have simulated the system by varying $m_{2}$ while keeping the other values constant. The graph shows the velocity and the postion as function of time. The graphs having a red triangle indicates the position $q_{2}$ and the other graphs the corresponding velocity $\dot{q}_{2}$. What we can see in the graph is that the object starts moving with an increasing velocity $\dot{q}_{2}$ while the acceleration $\ddot{q}_{2}$ decreases until a point where $\ddot{q}_{2}=0$. Thus, at this turning point the object starts to slow down until we have reached a stationary state where the velocity $\dot{q}_{2}=0$. This is the end point for the validity of our mathematical model based upon the assumptions we have made for the friction. What is also interesting to notice is that when $\ddot{q}_{2}=0$ there is not net force acting on the object, it's weightless. 


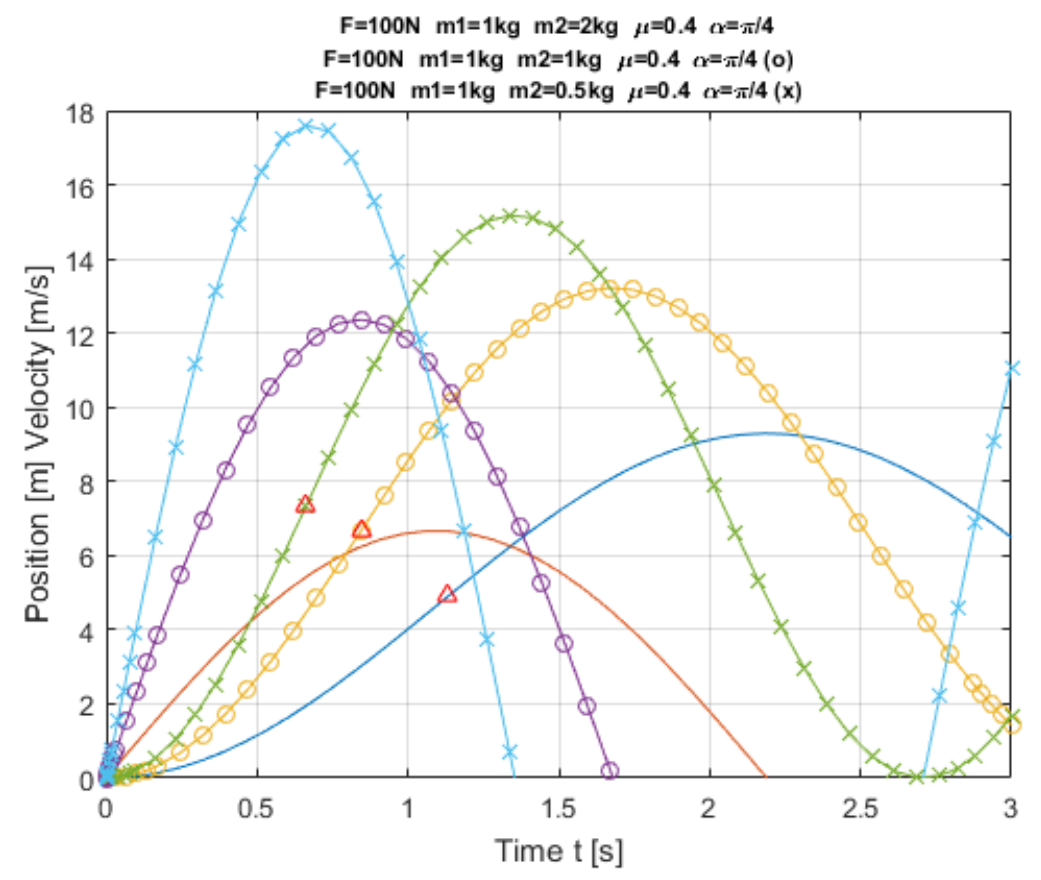

Figure 1:

By looking at these three graphs we can conclude that the increase of $m_{2}$ have a larger effect of the total time for the compression, thus $m_{2}=N \cdot m_{1}$ have larger effect on time than $m_{2}=m_{1} / N$. By comparing the compression of the spring we see that the change of $q_{2}$ between $2 \mathrm{~kg} \rightarrow 1 \mathrm{~kg}$ is roughly double that of $1 \mathrm{~kg} \rightarrow 0.5 \mathrm{~kg}$. One can understand that when $m_{2}$ is large the gravitation will be the dominant factor while for smaller $m_{2}$ the spring will have larger impact (the gravitation is independent of $q_{2}$ and the spring force dependent). Thus, decreasing $m_{2}$ will cause smaller and smaller differences in compression. While for higher $m_{2}$ we expect constant increasing changes. The next simulation we do is to see the effect of a changed friction force.

\subsubsection{Impact of variation in friction coefficient}

The results from the simulation can be seen in Figure 2 .

In this simulation all masses are the same and we can see that the difference in compression time is not affected as much as when increasing the mass of $m_{2}$. Also notice, compared to the previous simulation, in both cases we change the parameters in a linear fashion. This is due to the increasing inertia of $m_{2}$ taking longer time to accellerate. The total compression is decreasing with an increased friction, as expected, due to the loss of energy by means of heat. Let's estimate the relative loss of energy. By comparing $\mu=0.2$ which gives $\Delta W_{\text {pot }} \approx 1516 J$ and $\mu=0.4$ giving $\Delta W_{\text {pot }} \approx 567 J$ we are loosing approximately $\Delta W_{\text {heat }} \approx 949 \mathrm{~J}$. One might intuitively think that if we double the friction we 


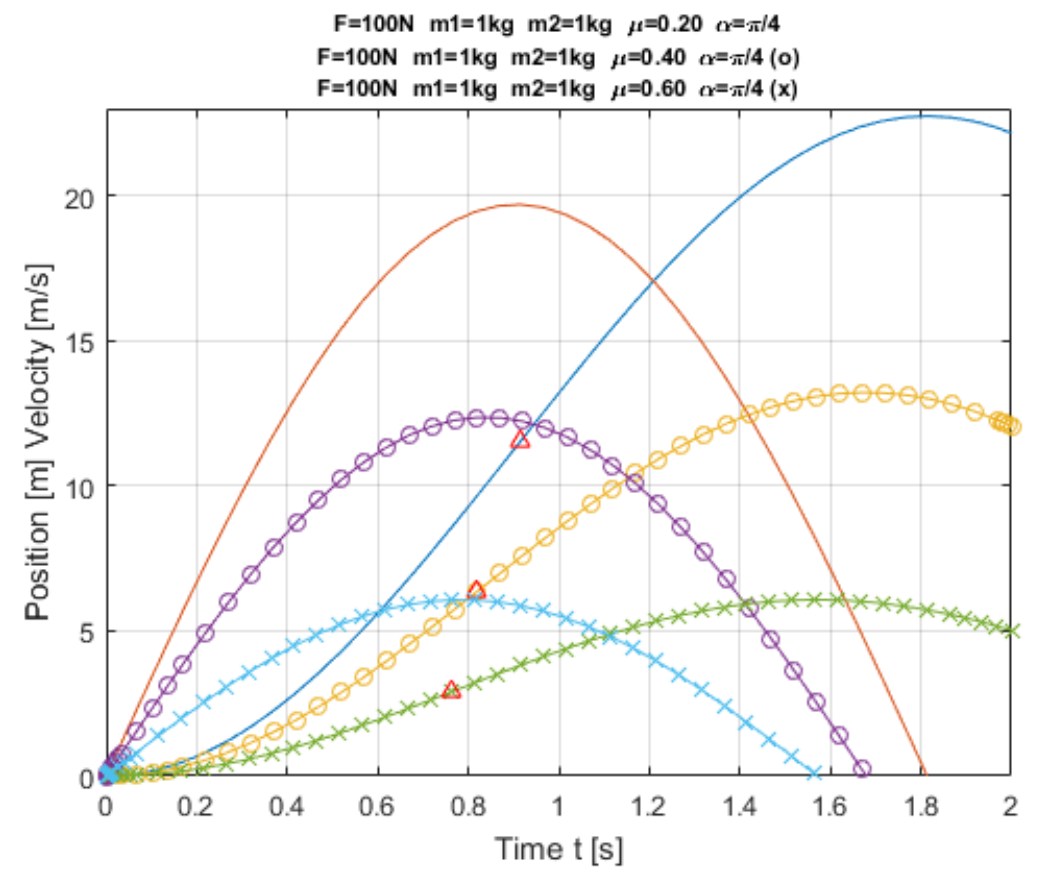

Figure 2:

would loose half of the originally available energy, thus $\Delta W_{\text {pot }} \approx 1516 \mathrm{~J} / 2=$ $758 \mathrm{~J}$. But instead we loose $25 \%$ more. This is not an insignificant number and in applications where friction can acctually be a problem we should really think about both the mechanical design and the mathematical model for the friction we use. In this paper, we have chosen a simple Coloumb friction. In the next simulation we increase the friction and will see the effect on the outcome of the mathematical model.

\subsubsection{Effect of an increased friction coefficient}

The results from the simulation can be seen in Figure 3. The graph can be compared with Figure 1.

In this graph we see that for the largest mass $m_{2}=2 \mathrm{~kg}$ we get both a negative velocity as well as position. And this is of course not physically valid, in this case it might be quite obvious how the system behaves in nature and it's quite easy to spot cases where the mathematical model might be questionable. In a more complicated technical system with many moving parts this might not always be the case. So the interpretation in this case is that the force $F=100 \mathrm{~N}$ is not large enough to overcome friction, gravity and spring force. The system is not moving. This example illustrates the importance of interpreting the results and understanding the validity of the mathematical model. 


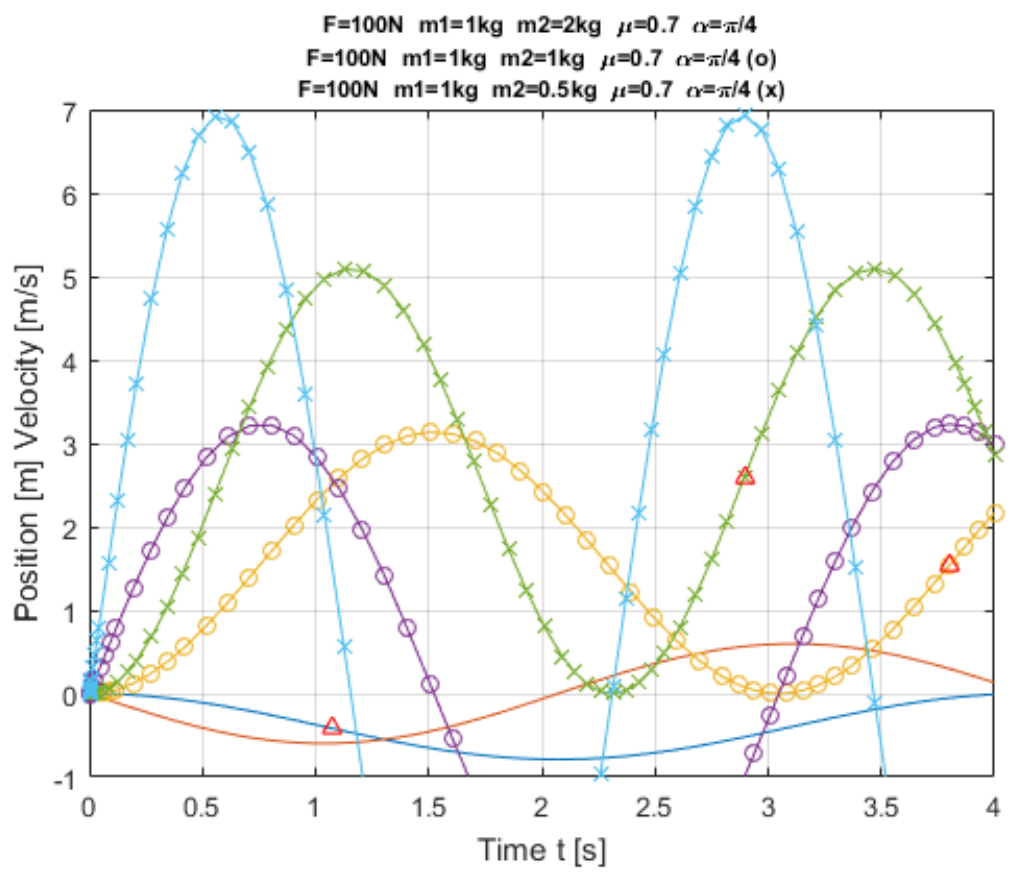

Figure 3:

\subsubsection{Impact of decreased wedge angle}

The last dynamic simulation that we will perform connects to the previous not moving system. Instead of choosing $\alpha=\pi / 4$ we instead decrease the angle of the wedge to $\alpha=\pi / 12$. Refer to Figure 4 and compare with Figure 3 .

What we can see in this result is that we once again have a moving system, by decreasing the angle for the wedge we can overcome the forces from the spring, friction and gravity. Comparing the total compression with Figure 3 we can also notice that in the previous case we loose a lot of energy to the friction which thus cannot be used for the compression.

\subsection{Static behaviour}

In the previous section we have looked at the dynamic behaviour of the system and we have interpreted the result of changing parameters in the mathematical model. And this analysis have led to an improved understanding about the system and maybe also some non-intuitive results. In comparison to pure physical understanding based upon human experience the mathematical model can provide us with results that are not intuitive and the one thing human experience cannot give us is a more or less accurate number. For some engineering applications the dynamics might be of interest while in others not. The original problem faced here was to get the spring constant of a fully compressed spring. This can be done by setting derivatives to zero in the equations of motion (7). We should however not disregard a dynamical simulation in more complicated 


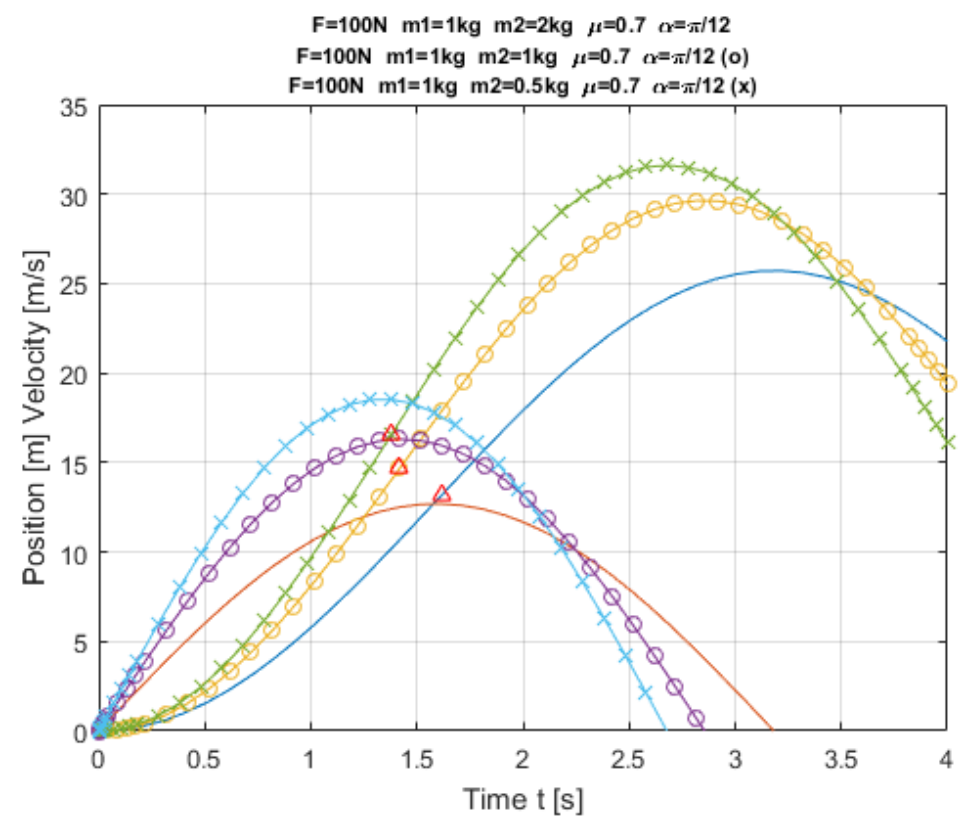

Figure 4:

models when there might be larger forces acting upon an object than in the stationary state. This might have influence for the strength of material in a situation where fatigue is important. In this paper someone might have been surprised over the weightless point of the spherical object when $\ddot{q}_{2}=0$.

The last analysis we will do is to see the effect of the friction for the force in the spring, and implicitly by knowing the spring constant $k$, the compression. The result from the simulation can be seen in Figure 5 .

In this graph we can see that for low friction coefficients the behaviour is getting transcendent for smaller angle $\alpha$. For larger friction coefficients the behaviour is more or less linear. If we loook at the expression

$$
k_{1} k_{2}=\frac{\cos (\alpha)-\mu \cdot \sin (\alpha)}{\sin (\alpha)+\mu \cdot \cos (\alpha)}
$$

we see that small $\mu$ mean that the behaviour goes as $\tan ^{-1}(\alpha)$ which approaches infinity when $\alpha \rightarrow 0$. For larger friction coefficients $\mu \cdot \sin (\alpha)$ will start having influence. By using this diagram we can easily choose a matching spring constant given the allowed compression. Also notice the independence of the wedge mass $m_{1}$.

\subsection{Final words}

Future work in this subject might include adding friction at the boundarys, e.g. given the force $F$ how much friction is needed for bringing the object into a stationary state and stay in that state when the force $F$ is removed. Is it even 


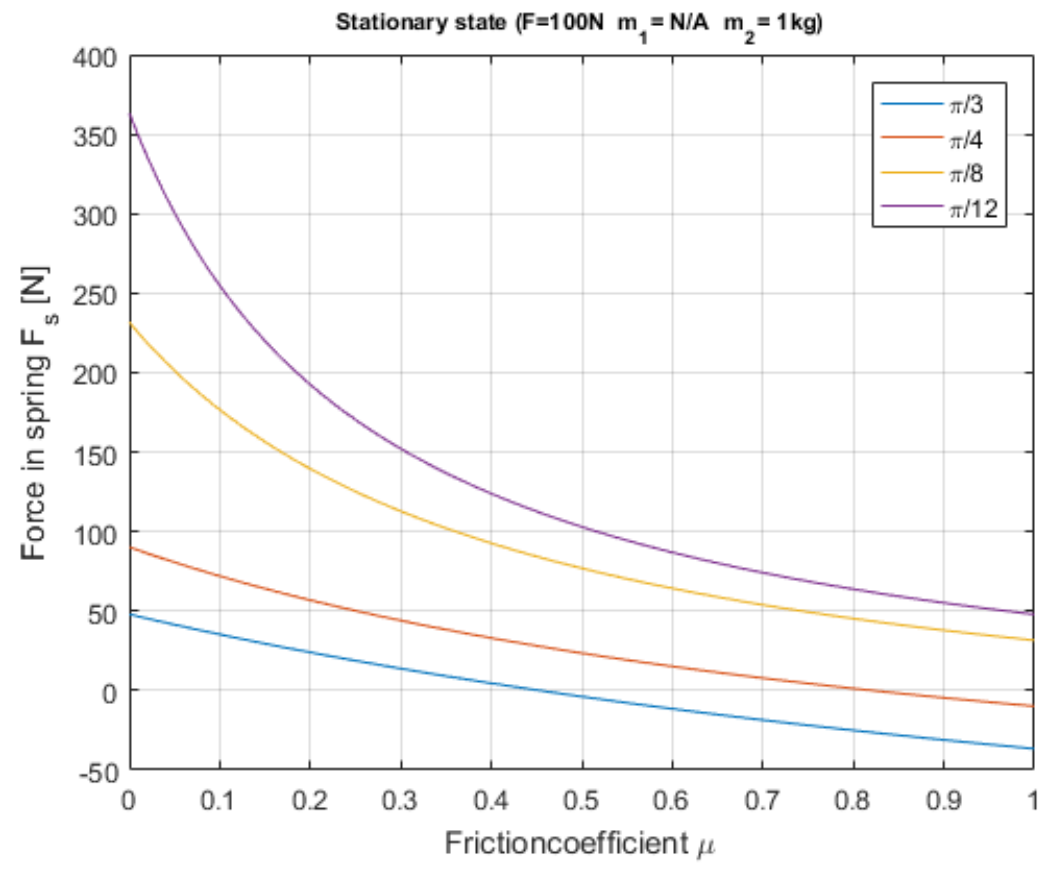

Figure 5:

possible. What is the result if choosing another friction model in Newtonian as well as in the Lagrangian approach. Adding values from real world example. Another application would be to include a thin layer of oil between the wedge and the sphere involving hydrodynamics. And this concludes this paper.

\section{References}

[1] Karl-Olof Olsson: Maskinelement. Liber, 2015.

[2] Leif Floberg. Maskinelement, Almqvist \& Wiksell Stockholm, 1971.

[3] Herbert Goldstein, Classical Mechanics, Addison-Wesley, 1980.

[4] E. Minguzzi, Rayleigh's dissipation function at work, arXiv, Cornell University 